\title{
Sensitivity and Specificity of Magnetic Resonance Cholangiopancreatography versus Endoscopic Ultrasonography against Endoscopic Retrograde Cholangiopancreatography in Diagnosing Choledocholithiasis: The Indonesian Experience
}

\author{
Dadang Makmun ${ }^{1}$, Achmad Fauzi ${ }^{1}$ and Hamzah Shatri ${ }^{2}$ \\ ${ }^{1}$ Division of Gastroenterology, ${ }^{2}$ Continuing Medical Education-Continuing Professional Development Unit, Department of Internal Medicine, \\ Faculty of Medicine Universitas Indonesia/Cipto Mangunkusumo National General Hospital, Jakarta, Indonesia
}

Background/Aims: Biliary stone disease is one of the most common conditions leading to hospitalization. In addition to endoscopic retrograde cholangiopancreatography (ERCP), endoscopic ultrasonography (EUS) and magnetic resonance cholangiopancreatography (MRCP) are required in diagnosing choledocholithiasis. This study aimed to compare the sensitivity and specificity of EUS and MRCP against ERCP in diagnosing choledocholithiasis.

Methods: This retrospective study was conducted after prospective collection of data involving 62 suspected choledocholithiasis patients who underwent ERCP from June 2013 to August 2014. Patients were divided into two groups. The first group (31 patients) underwent EUS and the second group (31 patients) underwent MRCP. Then, ERCP was performed in both groups. Sensitivity, specificity, and diagnostic accuracy of EUS and MRCP were determined by comparing them to ERCP, which is the gold standard. Results: The male to female ratio was 3:2. The mean ages were 47.25 years in the first group and 52.9 years in the second group. Sensitivity, specificity, accuracy, positive predictive value, and negative predictive value for EUS were $96 \%, 57 \%, 87 \%, 88 \%$, and $80 \%$ respectively, and for MRCP were $81 \%, 40 \%, 68 \%, 74 \%$, and $50 \%$, respectively.

Conclusions: EUS is a better diagnostic tool than MRCP for diagnosing choledocholithiasis. Clin Endosc 2017;50:486-490

Key Words: Endosonography; Cholangiopancreatography, magnetic resonance; Cholangiopancreatography, endoscopic retrograde; Choledocholithiasis

\section{INTRODUCTION}

Biliary stone disease is one of the most common diseases leading to hospitalization. However, it is difficult to determine the prevalence of gallstones in the general population because biliary stone disease is often asymptomatic. Only one-third of

Received: November 18, 2016 Revised: December 12, 2016 Accepted: December 12, 2016

Correspondence: Dadang Makmun

Division of Gastroenterology, Department of Internal Medicine, Faculty of Medicine Universitas Indonesia/Cipto Mangunkusumo National General Hospital, Jl. Diponegoro No. 71, Jakarta Pusat 10430, Indonesia

Tel: +62-21-3143957, Fax: +62-21-3142454, E-mail: hdmakmun@yahoo.com

(cc) This is an Open Access article distributed under the terms of the Creative Commons Attribution Non-Commercial License (http://creativecommons.org/ licenses/by-nc/3.0) which permits unrestricted non-commercial use, distribution, and reproduction in any medium, provided the original work is properly cited. gallstones cause symptoms or complications, such as choledocholithiasis. $^{1-3}$

A study in 2007 among patients who underwent endoscopic retrograde cholangiopancreatography (ERCP) associated with obstructive jaundice showed that choledocholithiasis is the most common cause of obstructive jaundice (54\%). It also causes tumors in papilla of Vater (17\%), pancreatic head tumor (13\%), common bile duct (CBD) stricture (5\%), cholangiocarcinoma (2\%), Klatskin tumor (2\%), and unidentified etiology (7\%). ${ }^{4}$

Among patients with symptomatic gallstones indicated for cholecystectomy, accurate preoperative detection of choledocholithiasis is required to decrease the operative risk and health care costs. ${ }^{5}$ Multiple modalities are available to confirm the diagnosis of choledocholithiasis, including clinical symp- 
toms, laboratory findings, transabdominal ultrasonography (TUS), computed tomography (CT), magnetic resonance cholangiopancreatography (MRCP), endoscopic ultrasonography (EUS), and ERCP. Intraoperative cholangiography (IOC) during cholecystectomy can also be used to diagnose choledocholithiasis.

The sensitivity and specificity of imaging modalities are important in the diagnosis of choledocholithiasis. Imaging modalities should be able to detect the presence of small stones in the bile duct. Despite the high sensitivity of TUS for diagnosing cholelithiasis, identifying choledocholithiasis with TUS is difficult. CT has a greater sensitivity than TUS for diagnosing choledocholithiasis, but the level of radiation and cost have limited its use as the first-line tool for diagnosing choledocholithiasis. Nonsurgical imaging modalities including MRCP, EUS, and ERCP provide true visualization of choledocholithiasis with comparable sensitivities. ${ }^{1}$

In the past decades, ERCP has become the gold standard for the diagnosis of choledocholithiasis, especially in patients with biliary tract obstruction that cannot be detected by TUS alone. Although ERCP is the gold standard for diagnosing choledocholithiasis, this procedure is highly dependent on operator skill and experience, and is associated with several complications such as pancreatitis, cholangitis, bleeding, and bowel perforation. A meta-analysis summarized the results of 21 prospective studies and found an average prevalence of post-ERCP pancreatitis (PEP) of $3.5 \%$ (range, $1.6 \%-15.7 \%$ ). Other complications of ERCP include bleeding (1.3\%), perforation $(0.1 \%-0.6 \%)$, cholangitis $(1 \%)$, and cholecystitis $(0.2 \%-0.5 \%)$. In addition, the percentage of deaths occurring as a consequence of any complication of ERCP has been reported to be $0.33 \%{ }^{5,6} \mathrm{~A}$ higher rate of PEP of $26.5 \%$ was reported in a prospective study conducted in our hospital. ${ }^{7}$ ERCP is reserved exclusively for therapeutic purposes because of the risks, and EUS and MRCP have become the modalities of choice for diagnosing choledocholithiasis. ${ }^{1-3}$

$\mathrm{MRCP}$ is a widely used noninvasive imaging technique for the evaluation of choledocholithiasis usually associated with CBD dilatation, and its diagnostic results are similar to those of ERCP. However, MRCP is used only in patients with clear indications because of its limitations. $\mathrm{CBD}$ dilatation can have diverse causes. Therefore, once MRCP demonstrates only a mildly dilated CBD, the physician is faced with the decision whether to investigate further using ERCP or to stop all investigations under the presumption that the dilatation reflects a normal variant. Despite improvements in MRCP techniques for imaging biliary abnormalities, its role is limited by the need for contrast agents and the inability to provide a histological diagnosis. Thus, EUS has emerged as an important tool for evaluating biliary disease. EUS can provide the opportu- nity to take biopsy samples for histopathologic examination and to determine the invasion and local staging of malignant lesions. EUS is also considered to be a superior diagnostic modality for evaluating unexplained CBD dilatation whose cause is inconclusive with MRCP. The increasing availability of EUS in hospitals and the greater diagnostic accuracy compared with MRCP suggest that EUS should be considered in the management of choledocholithiasis. ${ }^{3,5,8,9}$

The aim of this study was to compare the performance between EUS and MRCP in terms of the accuracy, sensitivity, and specificity for diagnosing choledocholithiasis, with ERCP as the gold standard.

\section{MATERIALS AND METHODS}

\section{Patient recruitment}

We consecutively enrolled 62 patients who underwent ERCP for suspected choledocholithiasis in our hospital from June 2013 to August 2014. The diagnosis was based on symptoms (most patients complained of upper right abdomen or epigastric pain and jaundice), laboratory findings (elevated liver enzymes especially alkaline phosphatase and gamma-glutamyl transferase, elevated bilirubin level), and abdominal ultrasonography examination (most patients were found to have dilated extrahepatic and intrahepatic bile ducts due to suspected choledocholithiasis). Patients with suspected sludge on the CBD were excluded. The patients were divided into two groups alternatingly; 31 patients underwent EUS and 31 patients underwent MRCP. After EUS or MRCP were performed, the patients underwent ERCP. The data were obtained from case report forms completed during the procedure.

\section{Methods}

All patients suspected of choledocholithiasis were diagnosed based on symptoms, laboratory findings, and ultrasonography results. Hematologic and biochemical tests were performed on all patients shortly after admission. TUS was performed on all patients by an expert ultrasonographer. On the first group, $\mathrm{MRCP}$ was performed using a $1.5 \mathrm{~T}$ magnetic resonance imaging (MRI) system (General Electric Medical System, USA), in which no medication or contrast medium was administered. On the second group, EUS was performed using a radial scope (Olympus Co., Japan) with a frequency of 6-7.5 MHz. ERCP was performed with a standard duodenoscope (TJF 180, Olympus Co., Japan) and a 1:1 diluted contrast medium.

\section{Data collection and analysis}

Patients aged 18-65 years who were diagnosed with cho- 
ledocholithiasis on the basis of clinical symptoms and radiological findings and who underwent either EUS or MRCP followed by ERCP were included in this study. Patients who refused to take part in this study and patients with contraindications for ERCP, such as hemodynamic instability, were excluded. The sensitivity, specificity, and diagnostic accuracy of EUS and MRCP were determined for the diagnosis of choledocholithiasis. The gold standard for the diagnosis of choledocholithiasis was ERCP. All procedures were performed by experienced doctors.

The following were assessed before and after the procedure: demographic data; clinical symptoms such as abdominal pain, dyspepsia, jaundice, nausea, vomiting, and fever; ultrasonography findings; laboratory findings; and the results of EUS or MRCP and ERCP. Statistical analysis was performed using Statistical Package for Social Sciences software version 21.0 (IBM SPSS, IBM Corp., Armonk, NY, USA).

\section{Ethical considerations}

Ethical approval was granted by The Ethical Committee of our Institution, with a registered ethical approval number 084/H2.F1/ETIK/2013.

\section{RESULTS}

Most patients in this study were men, with a male to female ratio of 3:2. The mean age was 52.9 years (standard deviation [SD] 13.31) in the MRCP group and 47.26 years (SD $11.04)$ in the EUS group. EUS had higher sensitivity (96\%), specificity (57\%), and accuracy (87\%) compared to MRCP in diagnosing choledocholithiasis.

The positive predictive value (PPV) and negative predictive value (NPV) of EUS were $88 \%$ and $80 \%$, respectively. The PPV and NPV of MRCP were $74 \%$ and $50 \%$, respectively, which were lower than that of the EUS. Tables 1 and 2 present the diagnostic values for each procedure.

Among 26 patients in this study with suspected choledocholithiasis who underwent EUS examination, 23 patients were found to have choledocholithiasis using ERCP. This means that the EUS stone detection rate was $88 \%$. On the other hand, among 23 patients with suspected choledocholithiasis who underwent MRCP examination, 17 patients were found to have choledocholithiasis by ERCP. This means that the MRCP stone detection rate was $73.4 \%$.

\section{DISCUSSION}

Choledocholithiasis refers to the presence of gallstones
Table 1. EUS and MRCP Results against ERCP

\begin{tabular}{llrcc}
\hline \multirow{2}{*}{ Diagnostic tool } & & \multicolumn{2}{c}{ ERCP (Gold standard) } \\
\cline { 3 - 5 } & & Stone & No stone \\
\hline \multirow{2}{*}{ EUS } & Stone & 26 & 23 & 3 \\
& No stone & 5 & 1 & 4 \\
\multirow{2}{*}{ MRCP } & Stone & 23 & 17 & 6 \\
& No stone & 8 & 4 & 4 \\
\hline
\end{tabular}

ERCP, endoscopic retrograde cholangiopancreatography; EUS, endoscopic ultrasonography; MRCP, magnetic resonance cholangiopancreatography.

Table 2. Comparison of Diagnostic Values between EUS and MRCP

\begin{tabular}{|lcc|}
\hline Variables & EUS & MRCP \\
\hline Compared to ERCP & & \\
$\quad$ True positive & 23 & 17 \\
\hline True negative & 4 & 4 \\
\hline False positive & 3 & 6 \\
\hline False negative & 1 & 4 \\
\hline Diagnostic test & & \\
\hline Sensitivity & $96 \%$ & $81 \%$ \\
\hline Specificity & $57 \%$ & $40 \%$ \\
\hline Accuracy & $87 \%$ & $68 \%$ \\
\hline PPV & $88 \%$ & $74 \%$ \\
\hline NPV & $80 \%$ & $50 \%$ \\
\hline
\end{tabular}

ERCP, endoscopic retrograde cholangiopancreatography; EUS, endoscopic ultrasonography; MRCP, magnetic resonance cholangiopancreatographyl; PPV, positive predictive value; NPV, negative predictive value.

within the $\mathrm{CBD}$ that usually originate from the gall bladder and pass into the CBD. Sometimes, the signs and symptoms alone are not a good predictor of the presence of choledocholithiasis, and various examinations are needed to make the diagnosis. An accurate, minimally invasive, and safe method is required to diagnose choledocholithiasis. ${ }^{10,11}$

Choledocholithiasis can be evaluated using several modalities. TUS is usually the first investigation for patients with suspected biliary diseases because it is safe for almost all patients. Choledocholithiasis is often missed on TUS because it has a relatively low sensitivity $(15 \%-40 \%)$, although, its sensitivity is better for detecting CBD dilatation (77\%-87\%). MRCP and EUS are other reliable procedures used to evaluate choledocholithiasis and have few risks and complications. ${ }^{5,9}$

From the data, we can conclude that the ability of EUS to diagnose true positive patients with choledocholithiasis was higher than MRCP (sensitivity $96 \%$ vs. $81 \%$ ). EUS exam- 
ination was also better for diagnosing true negative patients than MRCP (specificity $50 \%$ vs. $40 \%$ ). The PPV of EUS was $85 \%$, which indicates an $85 \%$ probability of a patient with choledocholithiasis having positive diagnostic test results; its NPV was $80 \%$, which indicates an $80 \%$ probability of a patient without choledocholithiasis having negative diagnostic test results. These PPV and NPV results are greater than MRCP results (PPV 74\%; NPV 50\%). Thus, in our study, EUS was superior to MRCP for detecting choledocholithiasis, which was also confirmed using ERCP.

In this study, the specificity of EUS and MRCP were lower than their sensitivity and accuracy. This result could be because of the high number of true negative and false positive cases for both EUS and MRCP, which indicates that the ability of the examiner to diagnose choledocholithiasis using those two modalities still needs to be developed, especially for diagnosing the small stones of choledocholithiasis.

Alhayaf et al. studied 90 patients who underwent EUS for suspected biliary tract abnormalities and reported 100\% sensitivity and $96 \%$ specificity for EUS for diagnosing choledocholithiasis compared with ERCP as the gold standard. ${ }^{11}$ Eshghi and Abdi studied 30 patients with gallstones and suspected choledocholithiasis based on an abnormal serum liver test and CBD dilation $>7 \mathrm{~mm}$ on TUS who underwent MRCP followed by open cholecystectomy and IOC. ${ }^{12}$ They reported $81.8 \%$ sensitivity and $87.5 \%$ specificity for MRCP in detecting choledocholithiasis; the PPV was $94.7 \%$ and NPV was $63.3 \%{ }^{12}$

Freitas et al. reviewed multiple modalities used to detect choledocholithiasis including laboratory tests, TUS, EUS, CT, and MRCP. ${ }^{2}$ The specificity of EUS was $96 \%-100 \%$ and that of MRCP was $92 \%-100 \%$. ${ }^{2}$ These findings suggest that these two modalities should be considered comparable to ERCP, which is the gold standard for the diagnosis of choledocholithiasis. Compared with ERCP, it was difficult to detect choledocholithiasis caused by stones $<5 \mathrm{~mm}$ in diameter using MRCP. $^{3}$ MRCP is also an expensive procedure that requires significant expertise for interpretation and is not always available to health care providers. In this situation, EUS is considered as a superior diagnostic modality for evaluating unexplained CBD dilatation that is inconclusive with MRCP. Using EUS to detect small stones $(<5 \mathrm{~mm})$ is better because it can provide high-resolution images and offers the distinct advantage of dynamic imaging in real time compared with $\mathrm{MRCP}^{3,8,13}$

A study by Tozzi di Angelo et al. evaluated the sensitivity, specificity, and accuracy of EUS and TUS compared with ERCP in 100 patients with a diagnosis of extrahepatic biliary obstruction. ${ }^{14}$ The sensitivity and accuracy of EUS for diagnosing CBD dilatation were $84 \%$ and $83 \%$, respective- ly, which were higher than the values obtained with TUS ( $46 \%$ and $66 \%$, respectively). However, the specificity of the two methods did not differ significantly. Palmucci et al. prospectively studied MRCP and EUS in 45 patients with extrahepatic biliary dilatation as shown by TUS, and did not find significant differences between EUS and MRCP for diagnostic accuracy. ${ }^{15}$ MRCP had $88.9 \%$ diagnostic accuracy, $91.9 \%$ sensitivity, $75 \%$ specificity, $94.4 \%$ PPV, and $66.7 \%$ NPV; EUS had 93.3\% diagnostic accuracy, 97.3\% sensitivity, $75 \%$ specificity, $94.7 \%$ PPV, and $85.7 \%$ NPV. ${ }^{15}$ Both modalities were highly accurate in detecting CBD stones but EUS had a slightly greater accuracy than MRCP. These results are similar to those reported by Aubé et al. and McMahon, who concluded that in patients with suspicion of mild to moderate choledocholithiasis, the accuracies of MRCP and EUS are similar. ${ }^{16,17}$ Sotoudehmanesh et al. used EUS in patients with pancreatobiliary disease referred for ERCP because of inconclusive MRCP. ${ }^{18}$ They found that EUS was a useful modality for confirming the diagnosis in cases of inconclusive MRCP indicating pancreatobiliary disorders.

Systematic reviews have compared the use of EUS and MRCP for diagnosing choledocholithiasis and have found similar results. Verma et al. performed a systematic review of five randomized, prospective blinded trials comparing

Table 3. A Proposed Strategy to Assign Risk of Choledocholithiasis in Patients with Symptomatic Cholelithiasis Based on Clinical Predictors

\begin{tabular}{|c|c|}
\hline \multicolumn{2}{|l|}{ Predictors of Choledocholithiasis } \\
\hline \multicolumn{2}{|l|}{ Very strong } \\
\hline \multicolumn{2}{|l|}{ CBD stone on TUS } \\
\hline \multicolumn{2}{|l|}{ Clinical ascending cholangitis } \\
\hline \multicolumn{2}{|l|}{ Bilirubin $>4 \mathrm{mg} / \mathrm{dL}$} \\
\hline \multicolumn{2}{|l|}{ Strong } \\
\hline \multicolumn{2}{|c|}{ Dilated CBD on TUS ( $>6 \mathrm{~mm}$ with gallbladder in situ) } \\
\hline \multicolumn{2}{|c|}{ Bilirubin $1.8-4 \mathrm{mg} / \mathrm{dL}$} \\
\hline \multicolumn{2}{|l|}{ Moderate } \\
\hline \multicolumn{2}{|c|}{$\begin{array}{l}\text { Abnormal liver biochemical test not including bilirubin } \\
\text { concentration }\end{array}$} \\
\hline \multicolumn{2}{|l|}{ Age older than 55 years } \\
\hline \multicolumn{2}{|l|}{ Clinical gallstones pancreatitis } \\
\hline \multicolumn{2}{|c|}{$\begin{array}{l}\text { Assigning a likelihood of choledocholithiasis based on clinical } \\
\text { predictors }\end{array}$} \\
\hline Presence of any very strong predictor & High \\
\hline Presence of both strong predictors & High \\
\hline No predictors present & Low \\
\hline All other patients & Intermediate \\
\hline
\end{tabular}

CBD, common bile duct; TUS, transabdominal ultrasonography. Adapted from Maple et al. ${ }^{22}$ 
MRCP and EUS for the detection of choledocholithiasis, with ERCP or IOC as the gold standard. ${ }^{19}$ They found that EUS and MRCP had high diagnostic performance overall and that the performance did not differ significantly between the two methods. ${ }^{19}$ Giljaca et al., in another recent systematic review, also concluded that EUS and MRCP have similar diagnostic accuracy for identifying choledocholithiasis. ${ }^{20}$

Performing EUS and ERCP consecutively in the same session has been shown to be safer than performing ERCP in a separate session, as shown in a study conducted by Benjaminov et al. ${ }^{21}$ This study found that performing ERCP in a separate session postponed treatment and resulted in significant biliary complications (including cholangitis and pancreatitis) in $14 \%$ of patients. This is similar to MRCP, which is performed by a radiologist and is usually performed in a different location and often on a different day; it is therefore less convenient for patients. To offer a convenient treatment, especially for low-to-moderate risk patients, EUS should be considered when MRCP is negative in patients with a moderate or high pretest probability, and ERCP should be performed immediately after stones are detected by EUS while the patient remains sedated. American Society for Gastrointestinal Endoscopy (ASGE) Standards of Practice Committee described clinical predictors as a proposed strategy to assign risk of choledocholithiasis in patients with symptomatic cholelithiasis that should also be considered in patients with suspected choledocholithiasis as presented in Table $3 .^{22}$

Conflicts of Interest

The authors have no financial conflicts of interest.

\section{Acknowledgments}

This research was supported by Cipto Mangunkusumo National General Hospital, Jakarta.

The authors would like to thank the Chief Director of Cipto Mangunkusumo National General Hospital, Jakarta, for awarding a research grant in 2013 and financing all expenses required for this study. We would also like to thank Siti Rahma Indah Permatasari, Oke Dimas Asmara, and Kartika Juwita for their excellent assistance in this study.

\section{REFERENCES}

1. Smith ZL, Meiselman MS. Calculous biliary disease. In: Bope ET, Kellerman RD, eds. Conn's current therapy. Philadelphia (PA): Elsevier Saunders; 2013. p. 500-503.

2. Freitas ML, Bell RL, Duffy AJ. Choledocholithiasis: evolving standards for diagnosis and management. World J Gastroenterol 2006;12:31623167.

3. Vázquez-Sequeiros E, González-Panizo Tamargo F, Boixeda-Miquel D, Milicua JM. Diagnostic accuracy and therapeutic impact of endoscopic ultrasonography in patients with intermediate suspicion of choledocholithiasis and absence of findings in magnetic resonance cholangiography. Rev Esp Enferm Dig 2011;103:464-471.

4. Makmun D, Sajuthi D, Daldiyono D, Winarto A, Sulistiawati E. Effect of intravenous polyunsaturated fatty acids administration on gastric mucosal integrity in pig-tailed macaques with obstructive jaundice. The Indonesian Journal of Gastroenterology, Hepatology, and Digestive Endoscopy 2011;12:8-14.

5. Al-Jiffry BO, Elfateh A, Chundrigar T, et al. Non-invasive assessment of choledocholithiasis in patients with gallstones and abnormal liver function. World J Gastroenterol 2013;19:5877-5882.

6. ASGE Standards of Practice Committee, Anderson MA, Fisher L, et al. Complications of ERCP. Gastrointest Endosc 2012;75:467-473.

7. Makmun D, Abdullah M, Syam AF, Fauzi A. Post-ERCP pancreatitis and its related factors: a prospective study in Cipto Mangunkusumo national general hospital. Journal of Digestive Endoscopy 2015;6:163-168.

8. Rana SS, Bhasin DK, Sharma V, Rao C, Gupta R, Singh K. Role of endoscopic ultrasound in evaluation of unexplained common bile duct dilatation on magnetic resonance cholangiopancreatography. Ann Gastroenterol 2013;26:66-70.

9. Yusuf TE, Bhutani MS. Role of endoscopic ultrasonography in diseases of the extrahepatic biliary system. J Gastroenterol Hepatol 2004;19:243250.

10. Prachayakul V, Aswakul P, Bhunthumkomol P, Deesomsak M. Diagnostic yield of endoscopic ultrasonography in patients with intermediate or high likelihood of choledocholithiasis: a retrospective study from one university-based endoscopy center. BMC Gastroenterol 2014;14:165.

11. Alhayaf N, Lalor E, Bain V, McKaigney J, Sandha GS. The clinical impact and cost implication of endoscopic ultrasound on use of endoscopic retrograde cholangiopancreatography in a Canadian university hospital. Can J Gastroenterol 2008;22:138-142.

12. Eshghi F, Abdi R. Routine magnetic resonance cholangiography compared to intra-operative cholangiography in patients with suspected common bile duct stones. Hepatobiliary Pancreat Dis Int 2008;7:525528.

13. Kondo $\mathrm{S}$, Isayama $\mathrm{H}$, Akahane $\mathrm{M}$, et al. Detection of common bile duct stones: comparison between endoscopic ultrasonography, magnetic resonance cholangiography, and helical-computed-tomographic cholangiography. Eur J Radiol 2005;54:271-275.

14. Tozzi di Angelo I, Prochazka V, Holinka M, Zapletalova J. Endosonography versus endoscopic retrograde cholangiopancreatography in diagnosing extrahepatic biliary obstruction. Biomed Pap Med Fac Univ Palacky Olomouc Czech Repub 2011;155:339-346.

15. Palmucci S, Mauro LA, La Scola S, et al. Magnetic resonance cholangiopancreatography and contrast-enhanced magnetic resonance cholangiopancreatography versus endoscopic ultrasonography in the diagnosis of extrahepatic biliary pathology. Radiol Med 2010;115:732-746.

16. Aubé C, Delorme B, Yzet T, et al. MR cholangiopancreatography versus endoscopic sonography in suspected common bile duct lithiasis: a prospective, comparative study. AJR Am J Roentgenol 2005;184:55-62.

17. McMahon CJ. The relative roles of magnetic resonance cholangiopancreatography (MRCP) and endoscopic ultrasound in diagnosis of common bile duct calculi: a critically appraised topic. Abdom Imaging 2008:33:6-9.

18. Sotoudehmanesh R, Khatibian M, Ghadir MR, et al. Diagnostic accuracy of endoscopic ultrasonography in patients with inconclusive magnetic resonance imaging diagnosis of biliopancreatic abnormalities. Indian J Gastroenterol 2011;30:156-160.

19. Verma D, Kapadia A, Eisen GM, Adler DG. EUS vs MRCP for detection of choledocholithiasis. Gastrointest Endosc 2006;64:248-254.

20. Giljaca V, Gurusamy KS, Takwoingi Y, et al. Endoscopic ultrasound versus magnetic resonance cholangiopancreatography for common bile duct stones. Cochrane Database Syst Rev 2015;(2):CD011549.

21. Benjaminov F, Stein A, Lichtman G, Pomeranz I, Konikoff FM. Consecutive versus separate sessions of endoscopic ultrasound (EUS) and endoscopic retrograde cholangiopancreatography (ERCP) for symptomatic choledocholithiasis. Surg Endosc 2013;27:2117-2121.

22. ASGE Standards of Practice Committee, Maple JT, Ben-Menachem T, et al. The role of endoscopy in the evaluation of suspected choledocholithiasis. Gastrointest Endosc 2010;71:1-9. 